\title{
Tverberg's Theorem at 50: Extensions and
}

\section{Counterexamples}

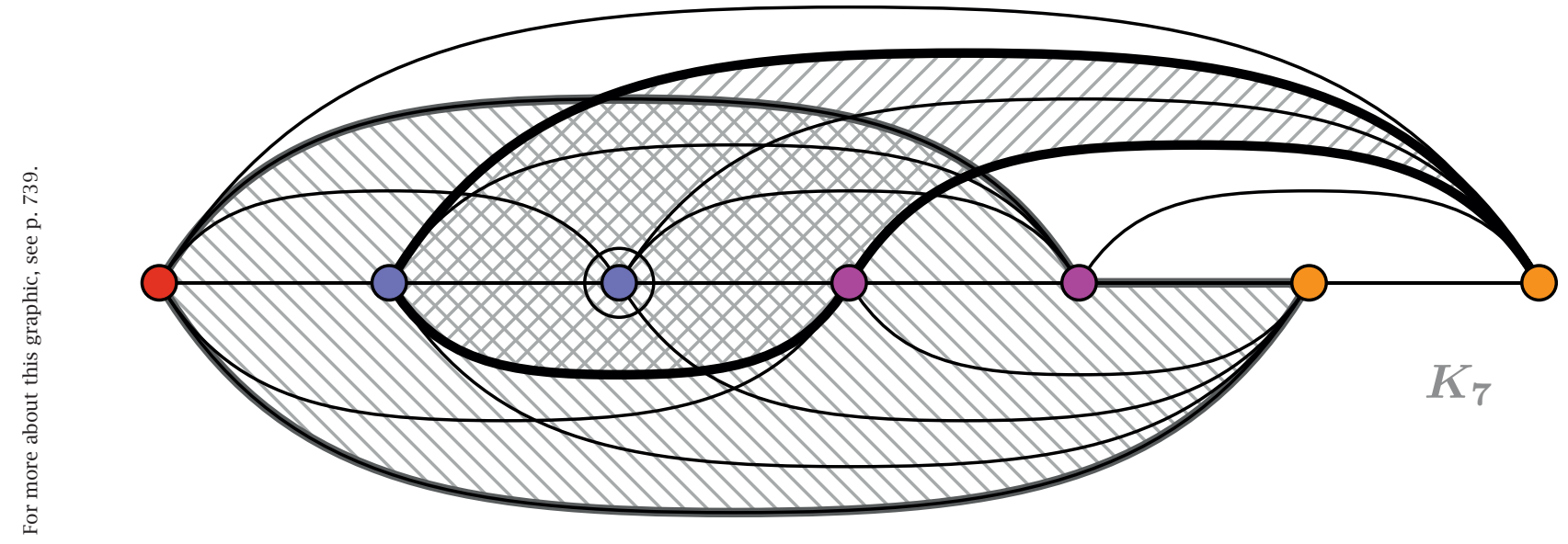

Imre Bárány, Pavle V. M. Blagojević, and Günter M. Ziegler

ABSTRACT. We describe how a powerful new "constraint method" yields many different extensions of the topological version of Tverberg's 1966 Theorem in the prime power caseand how the same method also was instrumental in the recent spectacular construction of counterexamples for the general case.

Imre Bárány is professor of mathematics at the Alfréd Rényi Institute, Budapest, and University College London. His email address is barany@renyi . hu.

Pavle V. M. Blagojević is a guest professor at the Berlin-Munich DFG Collaborative Research Center, "Discretization in Geometry and Dynamics," and a member of the Mathematical Institute of the Serbian Academy of Sciences and Arts in Belgrade. His email address is blagojevic@math. fu-berlin.de.

Günter M. Ziegler is professor of mathematics at Freie Universität Berlin. His email address is ziegler@math. fu-berlin.de. His work is supported by DFG via the Berlin Mathematical School and the CRC Discretization in Geometry and Dynamics.

For permission to reprint this article, please contact: reprint-permission@ams . org.

DOI: http://dx.doi.org/10.1090/noti1415
7 verberg's Theorem, whose fiftieth anniversary we celebrate this year, has played a central role both in discrete geometry and in topological combinatorics. The basic statement sounds easy:

Tverberg's Theorem (1966). Any $(d+1)(r-1)+1$ points in $\mathbb{R}^{d}$ may be grouped into $r$ disjoint subsets such that the convex hulls of these subsets have a point in common.

See Figure 1 for an example. The importance and impact of Tverberg's result - which the Norwegian mathematician Helge Tverberg proved in the early morning, freezing, in a hotel room in Manchester-may be seen from its many variations and extensions, among them its "colored" and "topological" versions. Curiously enough, the "topological Tverberg theorem," conjectured by the first author in 1976, has remained a conjecture for decades. The topological tools that we have for such problems, like the 1933 Borsuk-Ulam theorem, usually yield results only when $r$ is a prime power [13].

Now there are two surprising recent developments. First, with Florian Frick [2] we designed a "constraint method" that yields colored versions from the original 


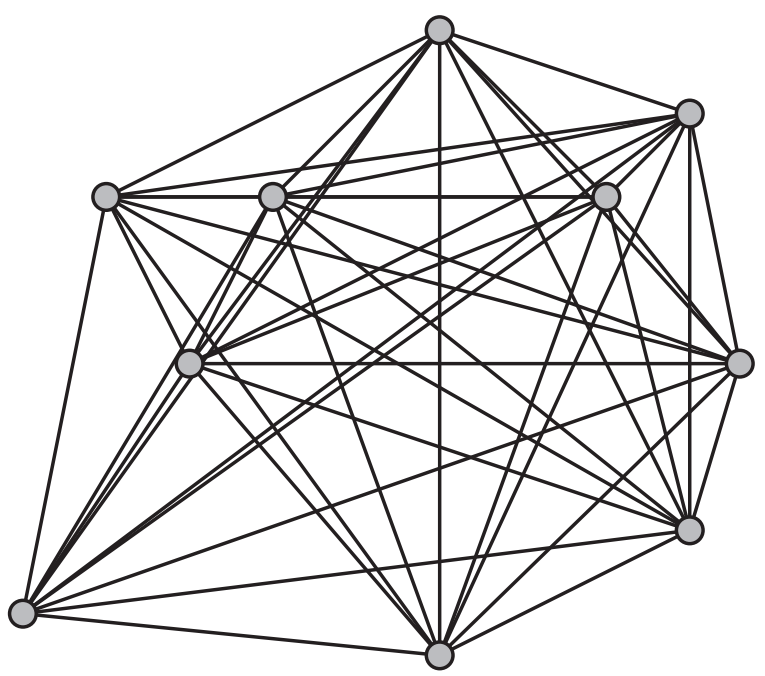

Figure 1. Tverberg's Theorem says that for $d=2$ and $r=4$, you can group these ten points into four disjoint subsets such that the convex hulls of these subsets have a point in common. See Figure 4 for a solution.

"topological Tverberg theorem" quite easily. Second, Isaac Mabillard and Uli Wagner in Vienna developed an " $r$ fold Whitney trick," and Florian Frick in Berlin noticed that combined with the constraint method this yields counterexamples for all $r \geq 6$ that are not prime powers.

This is big news, but major questions remain. For example, the current counterexamples to the topological Tverberg conjecture are in high dimensions. Does it also fail in the plane? Can one quantify the failure of the conjecture? In the prime power case, where the conjecture holds, are there always many Tverberg partitions? How can one find them? So many challenging questions remain. Tverberg's theorem and its variants will stay interesting and keep us busy into the future.

\section{Prehistory}

There are popular puzzles that lead one to discover that the graph $K_{5}$ of Figure 2 (the complete graph on five nodes and all ten pairs of nodes connected by an edge) is not planar; that is, the complete graph with five nodes cannot be drawn in the plane without intersections. This is a basic fact from graph theory and a starting point for the Four-Color Theorem, and it is not restricted to straight edges.

Indeed, in any drawing of the graph $K_{5}$ there are two nonadjacent edges that intersect. This also stays true beyond the usual "nice" drawings typically considered in graph theory, where edges might be drawn as straight lines or as smooth curves and will have at most a finite number of intersection points. It may be viewed as the special case $d=1$ of a famous result in topology, the Van Kampen-Flores theorem from 1932-33:

Any continuous map of the $d$-dimensional skeleton of a $(2 d+2)$-dimensional simplex $\Delta_{2 d+2}$ to $\mathbb{R}^{2 d}$ sends two points from disjoint faces of $\Delta_{2 d+2}$ to the same point in $\mathbb{R}^{2 d}$.

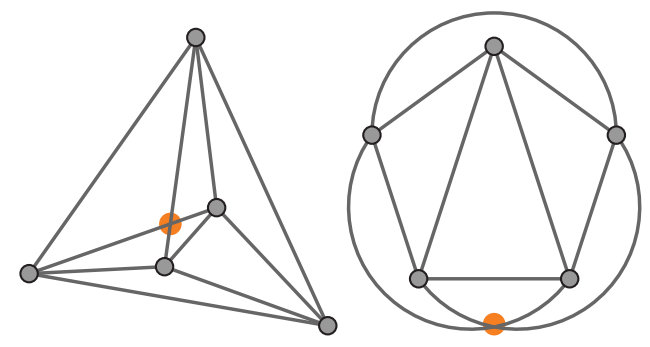

Figure 2. In every drawing of the complete graph $K_{5}$, whether by straight lines or by curves, there are two nonadjacent edges that intersect.

We will get back to this later, but our starting point here is even simpler, namely, drawings of $K_{4}$, the complete graph on four nodes, as in Figure 3. Here is a basic statement:

In every drawing of $K_{4}$, two nonadjacent edges intersect or one vertex is surrounded by the triangle spanned by the other three.

This seems quite obvious, and once again it has a high-dimensional version, Radon's theorem from 1921:

Any set of $d+2$ points in $\mathbb{R}^{d}$ contains two disjoint subsets whose convex hulls intersect.

This is easy to prove by linear algebra: If the points are $x_{1}, \ldots, x_{d+2}$ in $\mathbb{R}^{d}$, then the vectors $\left(x_{1}, 1\right), \ldots,\left(x_{d+2}, 1\right)$ are linearly dependent in $\mathbb{R}^{d+1}$; any linear dependence is given by reals $\lambda_{1}, \ldots, \lambda_{d+2}$ that are not all zero but sum to zero. So we can scale the dependence such that the positive $\lambda_{i}$ 's sum to 1 and the negative ones sum to -1 . This yields $\sum_{i: \lambda_{i}>0} \lambda_{i} x_{i}=\sum_{j: \lambda_{j}<0}\left(-\lambda_{j}\right) x_{j}$, which is what we need.

The result again can be phrased in terms of a map from a simplex to $\mathbb{R}^{d}$ :

Radon's Theorem. Any affine map $f: \Delta_{d+1} \rightarrow \mathbb{R}^{d}$ sends two points from disjoint faces of the $(d+1)$-simplex $\Delta_{d+1}$ to the same point in $\mathbb{R}^{d}$.

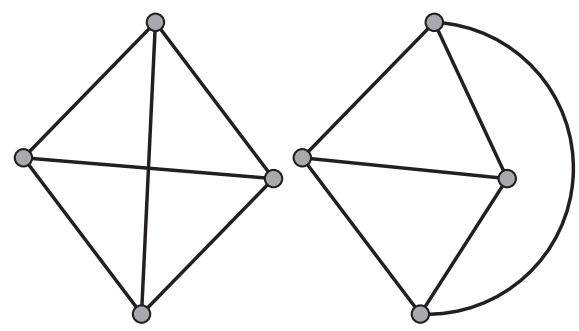

Figure 3. In every drawing of $K_{4}$, either two nonadjacent edges intersect or one vertex is inside the triangle spanned by the other three.

For $d=2$ this yields the statement about straight-line drawings of $K_{4}$. The conclusion is, however, true for very general drawings of $K_{4}$ in the plane given by any four points and six arbitrary continuous curves that connect them:

In every drawing of $K_{4}$, there are two nonadjacent edges that meet in the drawing, or there is one vertex surrounded by the cycle spanned by the remaining three vertices. 


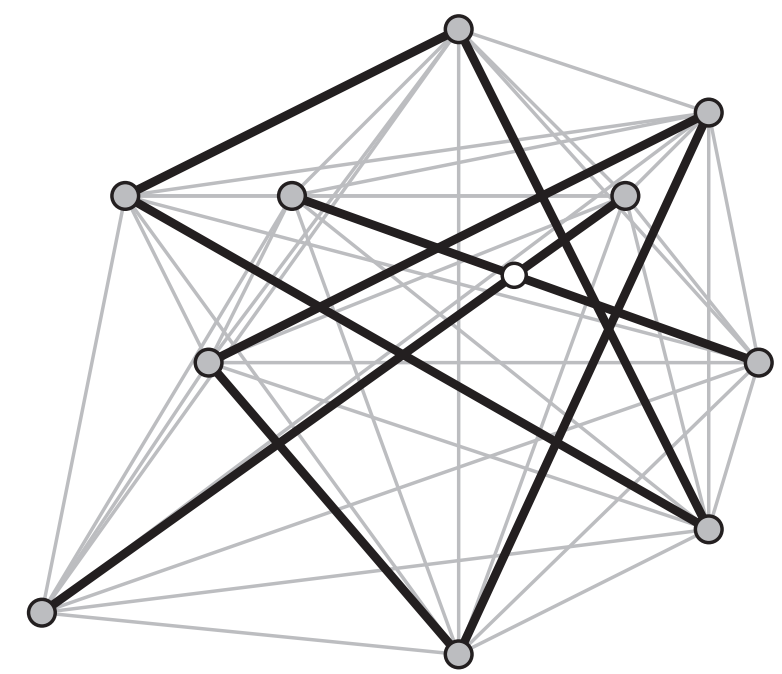

Figure 4. In this Tverberg partition for $r=4$, the marked intersection of two edges is contained inside the two bold triangles. This solves the puzzle at the beginning of the paper.

"Surrounded" would mean here that the three-edge cycle winds around the vertex, with nonzero winding number-interpret this in Figure 3!

How do we prove this? For $d=2$ this is easy to verify, since the drawing of $K_{4}$ in the plane without intersections is essentially unique. Nevertheless, if you work out details, you will need the Jordan curve theorem, which is less trivial than one might think. But again, there is a higherdimensional version, published by Ervin G. Bajmóczy and Imre Bárány in 1979:

The Topological Radon Theorem. Any continuous map $f: \Delta_{d+1} \rightarrow \mathbb{R}^{d}$ sends two points from disjoint faces of the $(d+1)$-simplex $\Delta_{d+1}$ to the same point in $\mathbb{R}^{d}$.

Bajmóczy and

Tverberg's theorem has been the most significant discovery in combinatorial convexity for the last fifty years.
Bárány derived their result from the BorsukUlam theorem, which in one of its many beautiful incarnations says that any continuous map $S^{d} \rightarrow \mathbb{R}^{d}$ sends two opposite points on the sphere to the same point in $\mathbb{R}^{d}$. (Gromov [7, p. 445] offers a particularly clever way of getting that: approximate the $(d+1)$-simplex by a strictly convex body, and map each point in the $d$-sphere to the unique point in the corresponding support hyperplane and then to $\mathbb{R}^{d}$; then apply the Borsuk-Ulam theorem to the corresponding map $S^{d} \rightarrow \mathbb{R}^{d}$.)
The topological Radon theorem was one of the starting points of what has since become the field of topological combinatorics. Indeed, the Borsuk-Ulam Theorem has an amazing range of applications in discrete geometry, combinatorics, and topology, as explained and illustrated in Matoušek's classic text Using the Borsuk-Ulam theorem.

On the other hand, the topological Radon theorem is the first step on the way to the topological Tverberg problem, which asks for multiple intersections in the case of continuous maps $\Delta_{N} \rightarrow \mathbb{R}^{d}$ for sufficiently large $N$. For affine maps this is the subject of Tverberg's theorem, whose fiftieth anniversary we are celebrating this year. For continuous maps it has been a mystery for decades, "one of the most challenging problems in this field" according to Matoušek; "a holy grail of topological combinatorics" according to Gil Kalai. Now it has been partially resolved, in an unexpected way, as we shall see.

\section{Tverberg's Theorem-Fifty Years Ago!}

In 1957 the Cambridge undergraduate Bryan John Birch proved, in his bachelor's thesis, an $r$-part version of Radon's result about straight-line drawings of $K_{4}$; it is illustrated by Figure 4 .

Birch's Theorem. In every straight-line drawing of $K_{3 r-2}$ there are two edges that cross such that the intersection point is contained in $r-2$ triangles spanned by disjoint triples of the other vertices, or there is one vertex of the graph contained in $r-1$ triangles formed by disjoint triples of the other vertices.

Again, this may be seen as a statement about affine maps, from the simplex $\Delta_{3 r-3}$ to the plane. Birch also studied a high-dimensional version of his result, but he couldn't prove it, so he finally published it as a conjecture and moved on to what he now calls "elliptic curvery" and became famous for a different conjecture, one of the million-dollar Clay Millennium Problems.

Meanwhile, a young Norwegian mathematician, Helge Tverberg, stumbled across the same problem, first re-proving it for the plane, then also for $\mathbb{R}^{3}$, and then, finally, on a cold early morning in a Manchester hotel room, establishing the general case. Tverberg phrased it as a statement about $(d+1)(r-1)+1$ points in $\mathbb{R}^{d}$, but again we prefer to state it in terms of affine

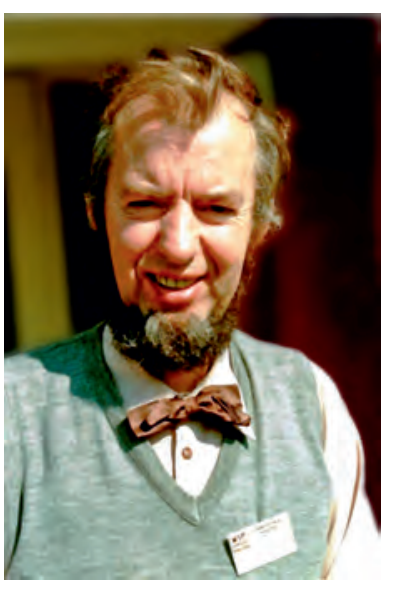

Helge Tverberg proved his theorem fifty years ago. This photo, by Gerard Sierksma, shows him in September 1985 at a conference in Pokrawna, Poland. maps.

Birch's Conjecture/Tverberg's Theorem. For integers $r \geq 2, d \geq 1$, and $N=(d+1)(r-1)$, any affine map $f: \Delta_{N} \rightarrow \mathbb{R}^{d}$ sends $r$ points from disjoint faces of the $N$-dimensional simplex $\Delta_{N}$ to the same point in $\mathbb{R}^{d}$. 
This is rather trivial for $d=1$; it is Birch's theorem for $d=2$ and Radon's theorem for $r=2$. Tverberg's original proof was complicated, showing that the statement remains true when the points move. It has been re-proved in new ways again and again, so it must be interesting.

Tverberg's theorem has been the most significant discovery in combinatorial convexity for the last fifty years. It has a great number of interesting applications in discrete and computational geometry, in combinatorics, and in theoretical computer science. Whenever you encounter a question about convexity properties of a finite point set in $\mathbb{R}^{d}$, check what Tverberg's theorem has to say.

The theorem also served as a model and inspiration for new results in combinatorial convexity, for instance the following beautiful one by Roman Karasev [9]: Given $3 r$ lines in general position in the plane, it is possible to split them intor disjoint sets each consisting of three lines such that the r triangles determined by the r triples of lines have a point in common. The result seems to extend to higher dimensions, but this partially remains a conjecture.

Tverberg's theorem prompted the start of several new research directions. In particular its topological and colored versions have created a large body of new knowledge and have led to novel connections between combinatorial convexity and algebraic topology, as we will now describe.

\section{The Topological Tverberg Conjecture-Forty Years Ago}

In 1976, forty years ago this year, the first author sent a letter to Helge Tverberg asking for a "topological version" of Tverberg's theorem. In May 1978 Tverberg presented the problem at the "Konvexe Körper" workshop in Oberwolfach, and it appears in the collection of problems distributed to the workshop participants (Figure 5).

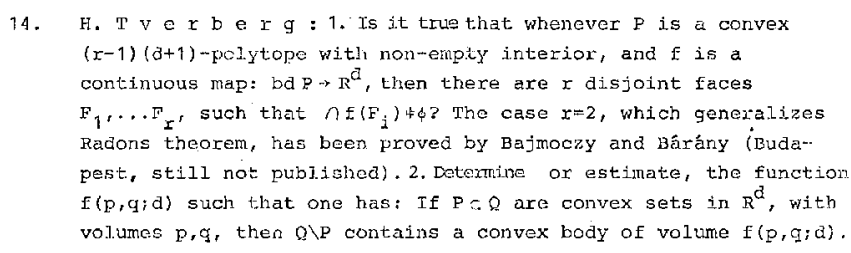

Figure 5. The topological Tverberg problem, in mimeographed notes from the 1978 Oberwolfach workshop, provided by Rolf Schneider.

The problem finally appeared in print in 1979. Since then it has been referred to as the "topological Tverberg conjecture" and often also, in acts of gross negligence, as the "topological Tverberg theorem."

The Topological Tverberg Conjecture [8]. For integers $r \geq 2, d \geq 1$, and $N=(d+1)(r-1)$, any continuous map $f: \Delta_{N} \rightarrow \mathbb{R}^{d}$ sends $r$ points from disjoint faces of the $N$-dimensional simplex $\Delta_{N}$ to the same point in $\mathbb{R}^{d}$.

We know that this conjecture holds for affine maps. For continuous maps, it was first proved by Imre Bárány, Senya B. Shlosman, and András Szűcs in 1981 [1]—only, however, under the unnatural-looking restriction that $r$ is a prime. How did this come in?

Sketch of the Proof for $r$ a Prime. Let us assume that there is a counterexample, that is, a continuous map $f: \Delta_{N} \rightarrow \mathbb{R}^{d}$ that does not send any collection of $r$ points from $r$ disjoint faces of the simplex $\Delta_{N}$ to the same point in $\mathbb{R}^{d}$. From the counterexample map $f$ one would be able to construct an " $r$-fold deleted product" map

$$
F=f^{\times r}:\left(\Delta_{N}\right)_{\Delta(2)}^{\times r} \rightarrow\left(\mathbb{R}^{d}\right)_{\Delta}^{\times r} \rightarrow S\left(W_{r}^{\oplus d}\right) \cong S^{N-r}
$$

Here $W_{r}:=\left\{\left(x_{1}, \ldots, x_{r}\right) \in \mathbb{R}^{r}: \sum x_{i}=0\right\}$. The point is that the symmetric group $\Im_{r}$ permutes the factors/coordinates for these spaces, and the map $F$ is "equivariant"; it respects the symmetry. Moreover, if $r$ is a prime, then a cyclic subgroup $\mathbb{Z}_{r} \subset \Im_{r}$ acts freely on the sphere, and thus one can apply an extension of the Borsuk-Ulam theorem for arbitrary free actions that is known as "Dold's theorem": It says that there is no equivariant map from an $(N-r)$-connected space to an $(N-r)$-dimensional one endowed with a free action. So we conclude that the counterexample map cannot exist.

So it's proved only if $r$ is a prime? To quote Matoušek: It seems likely that this theorem remains true for all $p$, not only primes, but so far nobody has managed to prove this. It has been verified for all prime powers, though.

Indeed, the next step was taken by Murad Özaydin in 1987. In an important and influential paper [12] that was never published, he proved the topological Tverberg conjecture for the case when $r=p^{k}$ is a prime power. How? He noted that in that case the hypothetical map

$$
F=f^{\times r}:\left(\Delta_{N}\right)_{\Delta(2)}^{\times r} \rightarrow\left(\mathbb{R}^{d}\right)_{\Delta}^{\times r} \rightarrow S\left(W_{r}^{\oplus d}\right) \cong S^{N-r}
$$

is equivariant with respect to an elementary abelian subgroup $\left(\mathbb{Z}_{p}\right)^{k} \subset \Im_{p^{k}}$ that acts on the sphere $S\left(W_{r}^{\oplus d}\right)$ not freely but without fixed points. In this situation some algebraic topology machinery (cohomology of the homotopy orbit space and a related localization theorem in combination with comparison of Serre spectral sequences) still implies that the map cannot exist. A detailed proof can be found in [3].

So it's proved only if $r$ is a prime power? Actually, Özaydin proved more: He showed that if $r$ is not a prime power, then the hypothetical map

$$
F:\left(\Delta_{N}\right)_{\Delta(2)}^{\times r} \rightarrow S^{N-r}
$$

does exist. This still doesn't mean that the topological Tverberg conjecture fails, but it means that the equivariant topology approach, known as the "Configuration Space Test Map Scheme (CS/TM)," fails here miserably. This was 
1987, nearly thirty years ago, and we were left without any promising approach or any hope of handling the topological Tverberg conjecture in the cases when the number of overlapping simplices is not a prime power.

\section{Using Constraints}

Over the years, there have been a great number of interesting extensions and variations of Tverberg's theorem. In every single case it seemed that one had to work hard, with major topological tools, to get the prime or even the prime-power case-and one could not get beyond that.

So it came as quite a surprise that there is a really simple "constraint method" to get virtually all of these extensions directly from the original topological Tverberg theorem. This observation arose in our collaboration [2] with Florian Frick at a blackboard at Arnimallee 2, a villa that is part of the Mathematical Institute of FU Berlin. We couldn't believe that it was so easy!

Let us illustrate the idea of the constraint method in two examples.

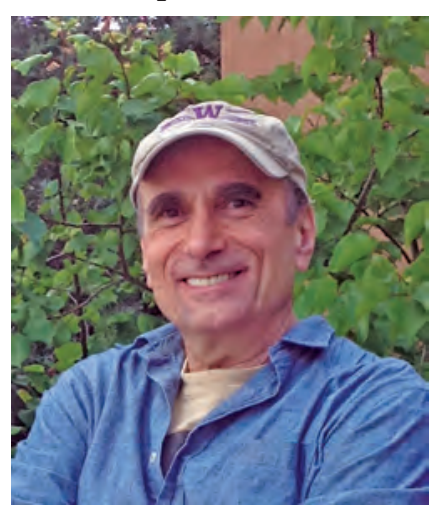

First, we demonstrate how the generalized Van Kampen-Flores theorem becomes a consequence of the topological Tverberg theorem: ${ }^{1}$

Theorem. If the topological Tverberg theorem holds for parameters $r$ and $d+1$, then the Generalized Van Kampen-Flores theorem holds for the parameters $r$ and $d$, for $N=(d+2)(r-1)$.

Murad Özaydin proved the topological Tverberg conjecture for the case when $r$ is a prime power.

The constraint method takes the extra condition that the Van Kampen-Flores theorem asks for and encodes it into a constraint function, which becomes an additional component of the Tverberg function, "one dimension higher." Any solution then will consist of $r$ faces of the simplex or, more precisely, points $x_{1}, \ldots, x_{r}$ on these faces, of equal distance from the skeleton. The pigeonhole principle forces one, and thus all, of the points to lie in the lower-dimensional skeleton:

Proof. Let $r \geq 2, d \geq 1, k \geq\left\lceil\frac{r-1}{r} d\right\rceil$ be integers, and let $N=(d+2)(r-1)$. Consider a continuous map $g: \Delta_{N} \rightarrow$ $\mathbb{R}^{d+1}$ defined by

$$
g(x)=\left(f(x), \operatorname{dist}\left(x, \operatorname{sk}_{k}\left(\Delta_{N}\right)\right)\right) .
$$

Here $\operatorname{sk}_{k}\left(\Delta_{N}\right)$ denotes the $k$-skeleton of the simplex $\Delta_{N}$. Since $N=((d+1)+1)(r-1)$ and the topological Tverberg theorem holds for $r$, it can be applied to the map $g$. Thus we get $r$ pairwise disjoint faces $\sigma_{i}$ of the simplex $\Delta_{N}$ with points $x_{i}$ in the relative interior of $\sigma_{i}$ such that $g\left(x_{1}\right)=$

${ }^{1}$ Later it was noted that Gromov had sketched this already in 2010, [7, pp. 445-446].
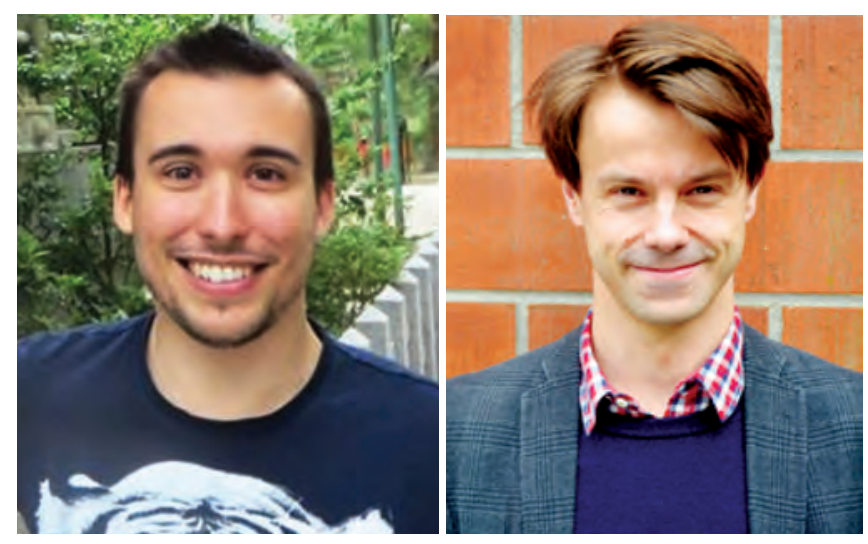

Isaac Mabillard and Uli Wagner developed the " $r$-fold Whitney trick" in the hope of constructing counterexamples.

$\cdots=g\left(x_{r}\right)$; that is, $f$ sends all points $x_{1}, \ldots, x_{r}$ to one point and in addition

$$
\operatorname{dist}\left(x_{1}, \operatorname{sk}_{k}\left(\Delta_{N}\right)\right)=\cdots=\operatorname{dist}\left(x_{r}, \operatorname{sk}_{k}\left(\Delta_{N}\right)\right) .
$$

Observe that if at least one of the faces $\sigma_{i}$ would belong to the $k$-skeleton, then $\operatorname{dist}\left(x_{i}, \operatorname{sk}_{k}\left(\Delta_{N}\right)\right)=0$ for all $1 \leq i \leq r$. Hence, it would follow that $\sigma_{i} \in \operatorname{sk}_{k}\left(\Delta_{N}\right)$ for all $1 \leq i \leq r$, and the proof of the theorem would be complete.

To conclude the proof we use the pigeonhole principle. Assume that all the faces $\sigma_{i}$ are not in the $k$-skeleton, that is, $\operatorname{dim} \sigma_{i} \geq k+1$ for every $i$. Since the faces $\sigma_{1}, \ldots, \sigma_{r}$ are disjoint they together have at least $r(k+2) \geq$ $r\left(\left\lceil\frac{r-1}{r} d\right\rceil+2\right) \geq N+2$ vertices, more than the number of vertices of the simplex $\Delta_{N}$, a contradiction.

Thus, if the generalized Van Kampen-Flores theorem does not hold for some parameter $r$, then the topological Tverberg theorem also does not hold for the same parameter.

Is this all? Can the pigeonhole principle create more results? Surprisingly enough, the answer is yes; it can give us much more. Maybe some colored Tverberg theorem?

Another Colored Tverberg Theorem. For integers $r \geq 1$, $d \geq 1$, and $N=(2 d+2)(r-1)$ where $r$ is a prime power, any continuous map $f: \Delta_{N} \rightarrow \mathbb{R}^{d}$, with any coloring of the vertex set of the simplex $\Delta_{N}$ by $d+1$ colors where each of the color classes is of size at most $2 r-1$, sends $r$ points from disjoint rainbow faces of the simplex $\Delta_{N}$-all of whose vertices must have different colors-to the same point.

Proof. Let $\left(C_{1}, \ldots, C_{d+1}\right)$ be a coloring of the set of vertices of the simplex $\Delta_{N}$ where $\left|C_{i}\right| \leq 2 r-1$ for all $i$. For each color class $C_{i}$ we introduce the subcomplex $\Sigma_{i}:=$ $\left\{\sigma \in \Delta_{N}:\left|\sigma \cap C_{i}\right| \leq 1\right\}$. Then the intersection $\Sigma_{1} \cap$ $\cdots \cap \Sigma_{d+1}$ is a subcomplex of all rainbow faces of $\Delta_{N}$. Let $g: \Delta_{N} \rightarrow \mathbb{R}^{2 d+1}$ be a continuous map defined by

$$
g(x)=\left(f(x), \operatorname{dist}\left(x, \Sigma_{1}\right), \ldots, \operatorname{dist}\left(x, \Sigma_{d+1}\right)\right) .
$$

With $r$ as a prime power and $N=((2 d+1)+1)(r-1)$, the topological Tverberg theorem can be applied to $g$. So, there are $r$ pairwise disjoint faces $\sigma_{i}$ with points 
$x_{i} \in$ relint $\sigma_{i}$ such that $g\left(x_{1}\right)=\cdots=g\left(x_{r}\right)$; that is, $f$ sends all points $x_{1}, \ldots, x_{r}$ to the same point and

$$
\begin{aligned}
\operatorname{dist}\left(x_{1}, \Sigma_{1}\right)= & \cdots=\operatorname{dist}\left(x_{r}, \Sigma_{1}\right), \\
& \cdots \\
\operatorname{dist}\left(x_{1}, \Sigma_{d+1}\right)= & \cdots=\operatorname{dist}\left(x_{r}, \Sigma_{d+1}\right) .
\end{aligned}
$$

For every subcomplex $\Sigma_{i}$ one of the faces $\sigma_{1}, \ldots, \sigma_{r}$ is contained in it. Indeed, if this were not true, then each of the faces $\sigma_{1}, \ldots, \sigma_{r}$ would have at least two vertices in the color class $C_{i}$. Since the faces are disjoint, the color classes $C_{i}$ have to be of size at least $2 r$, a contradiction. Thus, the distances that were previously equal have to vanish, implying that $x_{i} \in \sigma_{i} \subseteq \Sigma_{1} \cap \cdots \cap \Sigma_{d+1}$ for every $i$; that is, all the faces $\sigma_{1}, \ldots, \sigma_{r}$ are rainbow faces.

\section{Counterexamples}

Here comes the second surprise. From 2013 to 2015, Isaac Mabillard and Uli Wagner at IST Austria (in Klosterneuburg, near Vienna) developed an " $r$-fold Whitney trick" [10], which extends the classical Whitney trick designed for the embeddability problem (i.e., for $r=2$ ). Motivated by Özaydin's work, they had hoped to use this to construct counterexamples to the topological Tverberg conjecture. They proved the following essential theorem.

Theorem. For integers $r \geq 2, \ell \geq 3$, and an $((r-1) \ell)$ dimensional simplicial complex $K$, the following statements are equivalent:

(i) There exists an $\mathfrak{\Im}_{r}$-equivariant map $K_{\Delta(2)}^{\times r} \rightarrow S\left(W_{r}^{\oplus r \ell}\right)$.

(ii) There exists a continuous map $f: K \rightarrow \mathbb{R}^{r \ell}$ such that the $f$-images of any $r$ disjoint faces of $K$ do not intersect.

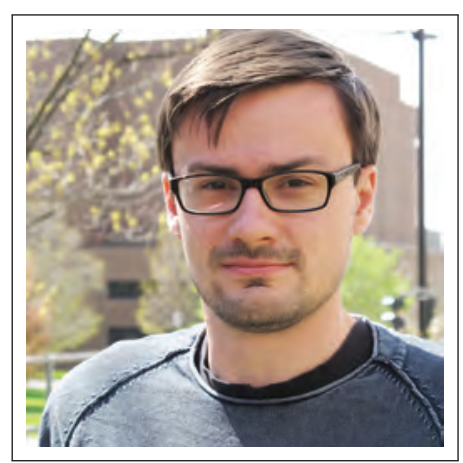

Florian Frick realized that the constraint method now yielded counterexamples to the topological Tverberg conjecture.

The "codimension three" condition that is classically inherent in the Whitney trick and is reflected in the statement of the theorem at the time prevented Mabillard and Wagner from obtaining counterexamples to the topological Tverberg theorem for nonprime powers. This was the state of affairs in January-February 2015, which Isaac Mabillard spent at the villa at FU Berlin, where he presented the progress from his PhD work in several

seminar lectures.

It turned out that only one more crucial observation was missing: Florian Frick, then a PhD student at TU Berlin and also a frequent visitor to the villa at FU Berlin, realized that the theorem of Mabillard and Wagner, in combination with the work of Özaydin and the constraint method, yields all that is needed for obtaining counterexamples. More precisely, counterexamples to the generalized Van Kampen-Flores conjecture for nonprime powers, which one could get from the Mabillard-Wagner theorem, would imply counterexamples to the topological Tverberg conjecture for nonprime powers [6], [3].

Theorem. Let $\ell \geq 3, r \geq 6$ be integers where $r$ is not $a$ prime power. For any integer $N>0$ there exists a continuous map $f: \Delta_{N} \rightarrow \mathbb{R}^{r \ell}$ such that the $f$-images of any $r$ disjoint faces from the $((r-1) \ell)$-skeleton of the simplex $\Delta_{N}$ do not intersect.

Sketch of the Proof. The deleted product cell complex $\left(\operatorname{sk}_{(r-1) \ell} \Delta_{N}\right)_{\Delta(2)}^{\times r}$ is a free $\mathfrak{s}_{r}$-space of dimension at most $d:=(r-1) r \ell$. Since $r$ is not a power of a prime, according to a result of Özaydin obtained via equivariant obstruction theory, there exists an $\Im_{r}$-equivariant map

$$
g:\left(\operatorname{sk}_{(r-1) \ell} \Delta_{N}\right)_{\Delta(2)}^{\times r} \rightarrow S\left(W_{r}^{\oplus d}\right) .
$$

The theorem of Mabillard and Wagner applied to the $\mathfrak{s}_{r^{-}}$ equivariant map $g$ yields a continuous map $f: \operatorname{sk}_{(r-1) \ell} \Delta_{N} \rightarrow$ $\mathbb{R}^{r \ell}$ with the property that the images of any $r$ disjoint faces from the $((r-1) \ell)$-skeleton of the simplex $\Delta_{N}$ do not intersect.

Thus we know that the generalized Van Kampen-Flores theorem fails for all $r$ that are not prime powers, and consequently the topological Tverberg conjecture fails for all such $r$. We also get explicit parameters for the counterexamples:

Corollary. Let $\ell \geq 3, r \geq 6$ be integers, and let $N:=$ $(r-1)(r \ell+2)$, where $r$ is not a prime power. There exists a continuous map $g: \Delta_{N} \rightarrow \mathbb{R}^{r \ell+1}$ such that $g$ images of any $r$ disjoint faces of the simplex $\Delta_{N}$ do not intersect.

The "smallest" counterexample to the topological Tverberg conjecture that can be obtained from this is (for $r=6$ ) a continuous map $f: \Delta_{100} \rightarrow \mathbb{R}^{19}$ such that the images of any six pairwise disjoint faces in $\Delta_{100}$ do not intersect.

In subsequent work, Mabillard and Wagner have improved this to In the cases where we know that a solution to Tverberg's theorem exists, is there any efficient way to find it?

in dimensions $3 r$ whenever $r$ is not a prime power, using a more elaborate tool ("prismatic maps") to overcome the codimension 3 restriction. More recently, together with Avvakumov and Skopenkov, they proved a codimension-2 analogue of the $r$-fold Whitney trick (for $r \geq 3$ ), leading to counterexamples in dimension $2 r$. Thus, the currently smallest counterexample (again for $r=6$ ) is a map $\Delta_{65} \rightarrow \mathbb{R}^{12}$. 


\section{The Next Fifty Years in the Life of Tverberg's Theorem?}

What's next? There are enough things to do! Let us point out four.

(1) The topological Tverberg conjecture for $d=2$ may be seen as a graph drawing problem for the complete graph $K_{3 r-2}$. Let's take $r=6$ : Is it true that in every drawing of $K_{16}$, there are two nonadjacent edges that meet in the drawing, with an intersection surrounded by four cycles spanned by disjoint triples of the remaining twelve vertices, or there is one vertex surrounded by five cycles spanned by the remaining fifteen vertices?

"Surrounded" here again means that the three-edge cycle winds around the point with nonzero winding number. One may get philosophical here: Is there an essential difference in the Tverberg-type properties between straight-line drawings and general drawings of graphs? For arrangements of lines vs. pseudolines in the plane, substantial combinatorial differences exist; Branko Grünbaum in 1970 had called this "the importance of being straight."

(2) Is it true in the affine case or if $r$ is a prime power that there are always many Tverberg partitions and not only one? Gerard Sierksma had conjectured in 1979 that there are always at least $(r-1) !^{d}$ solutions. Is this true? The best lower bound we have seems to be $\frac{1}{(r-1) !}(r / 2)^{(d+1)(r-1) / 2}$ for the prime case, by Vučić and Živaljević from 1993.

(3) In the cases where we know that Tverberg's theorem is true and a solution exists, is there any efficient way to find it? Is this (theoretically or practically) hard or easy? See Mulzer and Werner [11] for a discussion and recent references.

(4) The topological Tverberg theorem has an "optimal" colored extension [4] that has the color-free original version as a special case:

Optimal Colored Tverberg Theorem. For integers $r \geq 1$, $d \geq 1$, and $N=(d+1)(r-1)$ where $r$ is a prime, any continuous map $f: \Delta_{N} \rightarrow \mathbb{R}^{d}$ with any coloring of the vertex set of the simplex $\Delta_{N}$ where each color class is of size at most $r-1$ sends $r$ points from disjoint rainbow faces of the simplex $\Delta_{N}$ to the same point.

Note for this the concepts of "coloring" and "rainbow faces" have been redefined a bit: we use more than $d+1$ colors, and the rainbow faces do not pick up all colors. Among all the variations of the topological Tverberg theorem, this theorem is the only one that extends it, except that we proved it (together with Benjamin Matschke) only for the case where $r$ is prime, even in the case where the map is affine. Does it fail otherwise, say for $r=4$ and "ten colored points in the plane, no four of the same color," as in Figure 6?

\section{Acknowledgments}

The authors are grateful to Florian Frick, Andreas Holmsen, Isaac Mabillard, and Uli Wagner for very helpful and insightful comments on this paper.

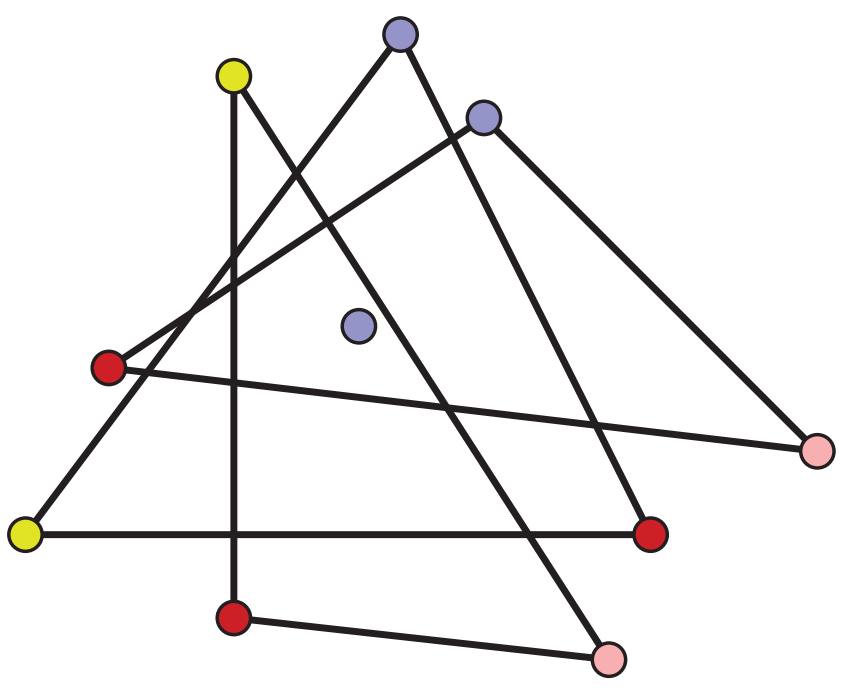

Figure 6. Does the optimal colored Tverberg theorem fail for nonprime $r$, say for $N+1=10$ colored points in the plane, no $r=4$ of the same color? This figure is not a counterexample, because the three rainbow triangles surround the point in the center.

\section{References}

[1] I. BÁrÁNY, S. B. SHLOSMAN, and A. SzŰCS, On a topological generalization of a theorem of Tverberg, J. Lond. Math. Soc. 23 (1981), 158-164. MR602247

[2] P. V. M. Blagojević, F. Frick, and G. M. Ziegler, Tverberg plus constraints, Bull. Lond. Math. Soc. 46 (2014), 953-967. MR3262197

[3] , Barycenters of polytope skeleta and counterexamples to the topological Tverberg conjecture, via constraints, preprint, 6 pages, October 2015, arxiv.org/abs/1510.07984.

[4] P. V. M. Blagojević, B. MAtschKe, and G. M. Ziegler, Optimal bounds for the colored Tverberg problem, J. Eur. Math. Soc. (JEMS) 17 (2015), 739-754. MR3336834

[5] P. V. M. Blagojević and G. M. ZiegleR, Beyond the BorsukUlam theorem: The topological Tverberg story, preprint, May 2016, 34 pages, arxiv.org/abs/1605.07321.

[6] F. FRICK, Counterexamples to the topological Tverberg conjecture, Oberwolfach Reports 12 (2015), 318-322.

[7] M. Gromov, Singularities, expanders and topology of maps. II: From combinatorics to topology via algebraic isoperimetry, Geom. Funct. Anal. (GAFA) 20 (2010), 416-526. MR2671284

[8] P. M. GRuber and R. Schneider, Problems in geometric convexity, in "Contributions to Geometry" (Proc. Geometry Symposium, Siegen, 1978), J. Tölke and J. Wills, eds., Birkhäuser, Basel, 1979, pp. 255-278. MR568503

[9] R. N. KARASEV, Dual theorems on central points and their generalizations, Sbornik Math. 199 (2008), 1459-1479. English translation of Russian original in Mat. Sb. 199, No. 10 (2008), 41-62. MR2473811

[10] I. MABILlard and U. WAgner, Eliminating highermultiplicity intersections, I. A Whitney trick for Tverbergtype problems, preprint, 46 pages, arxiv.org/abs/1508. 02349, August 2015.

[11] W. MulzER and D. WERNER, Approximating Tverberg points in linear time for any fixed dimension, Discrete Comput. Geom. 50 (2013), 520-535. MR3090531 
[12] M. ÖZAYDIN, Equivariant maps for the symmetric group, preprint, 17 pages, digita1.1ibrary.wisc.edu/1793/63829, 1987.

[13] G. M. ZIEGLER, 3N colored points in a plane, Notices Amer. Math. Soc. 58 (2011), 550-557. MR2807521

\section{Credits}

Photo of Imre Bárány, courtesy of Oberwolfach Photo Collection, (CMFO.

Photo of Pavle V. M. Blagojević, courtesy of Oberwolfach Photo Collection, CMFO.

Photo of Günter M. Ziegler, courtesy of Stefan Gräf.

\section{About the Opening Graphic}

The "Optimal Colored Tverberg Theorem" says-for $d=2$ and $r=3$-that any drawing of the complete graph $K_{7}$ on seven nodes, no three of the same color, either has two rainbow triangles covering the seventh node, or two rainbow edges that cross in a rainbow triangle spanned by the other three nodes, or both. Our drawing shows an example for the first case. How about the second one?

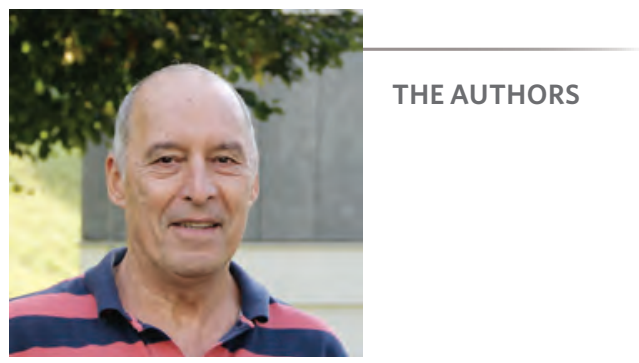

Imre Bárány

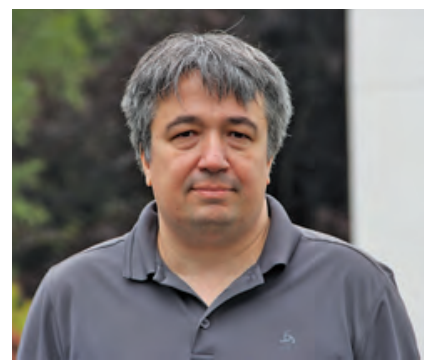

Pavle V. M. Blagojević

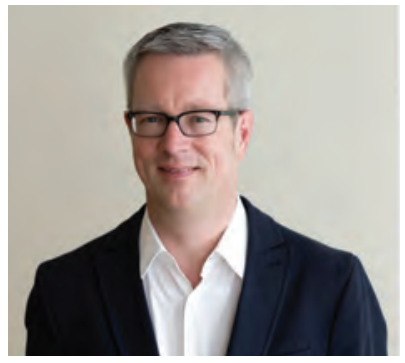

Günter M. Ziegler

\section{Let Your Voice Be Heard!}

\section{Do you enjoy what you're reading in the Notices?}

\section{Would you like to see an article on a certain topic? Person? Situation?}

Then we want to hear from you. Use our Notices Online Commentary Feature to post your general comments and suggestions:

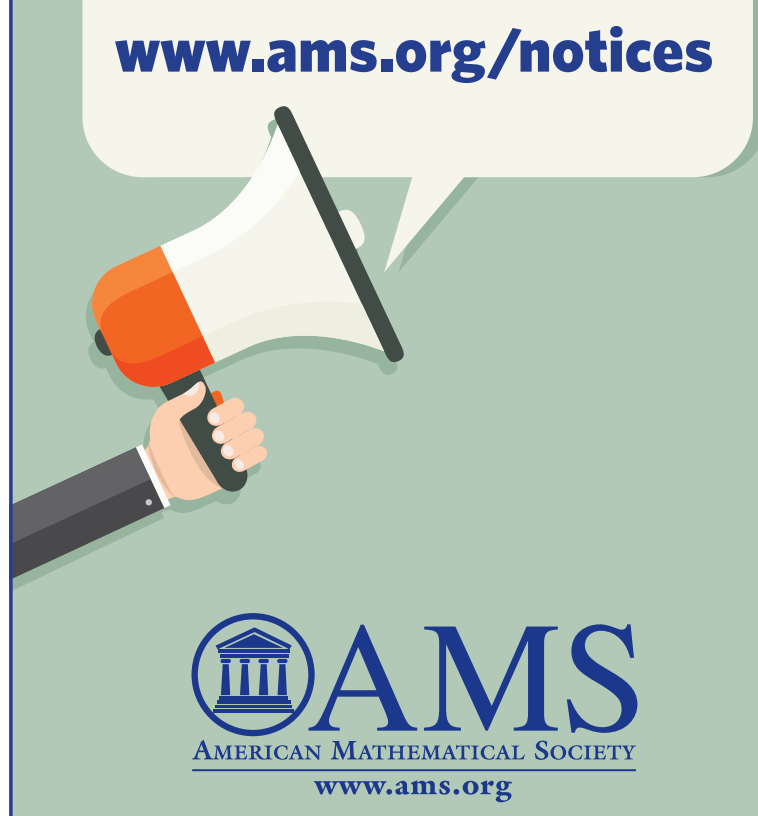

